\title{
Research Article \\ Oxidative Stress Level as a Predictor of Anastomotic Leakage after Rectal Surgery
}

\author{
Jiajun Luo, Hongxue Wu, Yu Yang, Yue Jiang, Jingwen Yuan, and Qiang Tong $\mathbb{D}$ \\ Department of Gastrointestinal Surgery I Section, Renmin Hospital of Wuhan University, Wuhan 430060, China \\ Correspondence should be addressed to Qiang Tong; qiangtong@whu.edu.cn
}

Received 4 April 2021; Accepted 4 June 2021; Published 29 June 2021

Academic Editor: Mingliang Jin

Copyright (C) 2021 Jiajun Luo et al. This is an open access article distributed under the Creative Commons Attribution License, which permits unrestricted use, distribution, and reproduction in any medium, provided the original work is properly cited.

\begin{abstract}
Background. Early diagnosis of anastomotic leakage (AL) after rectal surgery can reduce the adverse effects of AL, thereby reducing morbidity and mortality. Currently, there are no accepted indicators or effective scoring systems that can clearly identify patients at risk of anastomotic leakage. Methods. A prospective study with assessment of the diagnostic accuracy of oxidative stress level (CAT, SOD, MDA) in serum and drain fluid compared to white blood cell count (WBC), C-reactive protein (CRP), and neutrophil percentage (NEUT) in prediction of AL in patients undergoing elective rectal surgery with anastomosis. Results. Most of the oxidative stress indicators we detected are of considerable significance in the diagnosis of anastomotic leakage. The level of MDA on postoperative day (POD) 3 (areas under the curve (AUC): 0.831) and POD5 (AUC: 0.837) in the serum and on POD3 (AUC: 0.845 ) in the drain fluid showed the same excellent diagnostic accuracy as the level of CRP on the POD3 (AUC: 0.847) and POD5 (AUC: 0.896). Conclusions. The overall level of oxidative stress in serum and drain fluid is a reliable indicator for the early diagnosis of anastomotic leakage after rectal surgery. More specifically, among the redox indicators analyzed, MDA has almost the same predictive value as CRP, which provides another useful biomarker for the early diagnosis of anastomotic leakage.
\end{abstract}

\section{Introduction}

Colorectal cancer (CRC) is the third most commonly diagnosed malignancy and the fourth leading cause of cancerrelated deaths in the world [1]. It is widely accepted that colorectal cancer continues to be a severe problem. Colorectal surgery is long established as the mainstay treatment for colorectal cancer [2]. Rectal cancer accounts for about $67 \%$ of colorectal cancers and has a higher incidence of postoperative anastomotic leakage (AL) than colon surgery [3]. $\mathrm{AL}$ occurs in $4 \%-33 \%$ [4] of patients and is a major complication after restorative resection for rectal cancer that may adversely impact morbidity, mortality, and functional outcomes $[5,6]$. Despite efforts to reduce AL occurrence, the incidence of $\mathrm{AL}$ has remained relatively unchanged over the last several years [7].

The ongoing occurrence of $\mathrm{AL}$ is due to many factors including those pertaining to surgical technique such as blood supply, tension, suture type, or device deployment, as well as, patient-related factors such as frailty, poor nutritional status, or chemoradiotherapy [8]. AL is clearly associated with the healing process following surgery of the gut. Reactive oxygen species (ROS) and oxidative stress have long been recognized as key components in wound healing [9]. Furthermore, data have shown that the production of specific ROS and the activation of specific formyl peptide receptors (FPRs) regulate intestinal wound healing [10]. Low concentrations of ROS production are necessary to ward off invading microorganisms and are crucial for cell survival signaling, but excessive ROS or impaired ROS detoxification causes oxidative damage, which may lead to AL [11].

At present, the surgeons' clinical risk assessment has a low predictive value for AL in rectal surgery [12]. Early diagnosis of AL is important to allow for alternative treatments to prevent morbidity and mortality. Many scholars have made great efforts to find early AL diagnostic markers. Previous studies have reported that C-reactive protein (CRP) [1316], procalcitonin (PCT) [14], cytokines [17], lactate [18], and amylase [19] could help in the early diagnosis of AL, but limitations remain. Considering the important role of ROS in intestinal healing, we believe that the detection of ROS and oxidative stress levels could be a complementary 
TABLE 1: Characteristics of rectal cancer (RC) patients.

\begin{tabular}{lccc}
\hline Parameter & AL $(n=16)$ & Without AL $(n=254)$ & $P$ value \\
\hline Gender, M (\%) & $12(75.0)$ & $150(59.1)$ & 0.294 \\
Age, mean \pm s (years) & $61.3 \pm 10.3$ & & \\
Comorbidities, $n$ (\%) & & $13(5.1)$ & 0.608 \\
$\quad$ Metabolic & $3(18.8)$ & $52(20.5)$ & \\
$\quad$ Cardiovascular & $6(37.5)$ & $172(67.7)$ \\
ASA score, $n$ (\%) & & $82(32.3)$ \\
$1-2$ & $12(75.0)$ & $26(10.2)$ \\
3 & $4(25.0)$ & & 0.120 \\
Neo-adjuvant CRT, $n(\%)$ & $5(31.3)$ & $76(29.9)$ \\
Stage, TNM, $n(\%)$ & & $54(21.3)$ \\
I & $1(6.2)$ & $114(44.9)$ \\
II & $7(43.8)$ & $10(3.9)$ \\
III & $8(50.0)$ & 0.544 \\
IV & $0(0)$ & 0.070 \\
\hline
\end{tabular}

TABle 2: Comparison of CRP/WBC/NEUT on POD3 and POD5 in patients with and without anastomotic leak.

\begin{tabular}{lccc}
\hline Parameter $($ mean \pm SD) & AL $(n=16)$ & Without AL $(n=254)$ & $P$ value \\
\hline $\mathrm{CPR}_{\text {POD3 }}$ & $109.8 \pm 39.8$ & $65.2 \pm 22.3$ & $<0.001$ \\
$\mathrm{WBC}_{\text {POD3 }}$ & $9.492 \pm 4.063$ & $8.703 \pm 3.086$ & 0.332 \\
$\mathrm{NEUT}_{\text {POD3 }}$ & $84.02 \pm 5.847$ & $78.05 \pm 5.368$ & $<0.001$ \\
$\mathrm{CPR}_{\text {POD5 }}$ & $111.2 \pm 53.2$ & $43.5 \pm 18.4$ & $<0.001$ \\
$\mathrm{WBC}_{\text {POD5 }}$ & $11.054 \pm 3.844$ & $7.723 \pm 2.477$ & $<0.001$ \\
NEUT $_{\text {POD5 }}$ & $82.72 \pm 10.96$ & $74.01 \pm 8.798$ & $<0.001$ \\
\hline
\end{tabular}

CRP: C-reactive protein; NEUT: neutrophil percentage; WBC: white blood cells; POD: postoperative day.

method for the early diagnosis of AL. In this study, the levels of three redox indicators, catalase (CAT), malondialdehyde (MDA), and superoxide dismutase (SOD) will be determined in patients' serum and drain fluid and assessed for correlation with AL. We will also measure C-reactive protein (CRP) along with quantification of white blood cells (WBC) and neutrophil percentage (NEUT) for comparison to determine its diagnostic accuracy.

\section{Materials and Methods}

2.1. Patients. All patients were diagnosed with rectal cancer and underwent elective rectal surgery with primary anastomosis. The exclusion criteria included patients under 18 years of age, emergency operations, advanced cancers that were not amenable to curative resection, immunosuppression, and patients with severe infections or an American Society of Anesthesiologists (ASA) physical status $\geq 4$. Preoperative workup included a physical examination, colonoscopy, computed tomography (CT), and chest X-ray or chest CT. The decision to perform laparoscopic or open surgery is based on the patient's condition and willingness. The diagnosis of AL is based on intestinal contents in the peritoneal drainage fluid and oral contrast agent exudation during fluo- roscopy or CT examination. The present study was designed as a prospective observational pilot study. Written informed consent was obtained from all patients prior to enrolment. This study was approved by the Ethics Committee of Renmin Hospital of Wuhan University.

2.2. Analytical Methods. Blood samples were collected from patients on postoperative day (POD) 3 and POD5. Blood was drawn by venipuncture, allowed to clot for 30 minutes, and then centrifuged $(10 \mathrm{~min}, 1000 \times \mathrm{g})$. Serum was collected and kept frozen at $-80^{\circ}$ until examination. Drain fluid were collected on POD3 and POD5 and kept frozen at $-80^{\circ}$ until examination. The quantification of the WBC and NEUT was performed with a hematology analyzer. The C-reactive protein (CRP) concentration in serum was determined by the turbidimetric method with a CRP test. Catalase (CAT), malondialdehyde (MDA), and superoxide dismutase (SOD) levels in serum and drain fluid were determined using commercially available kits (Nanjing Jiancheng Bioengineering Institute, Nanjing, China).

2.3. Statistical Analysis. Statistical analyses were performed using SPSS Statistics 22.0 software. $P$ values $<0.05$ were considered statistically significant. The $\chi^{2}$ test for independence 


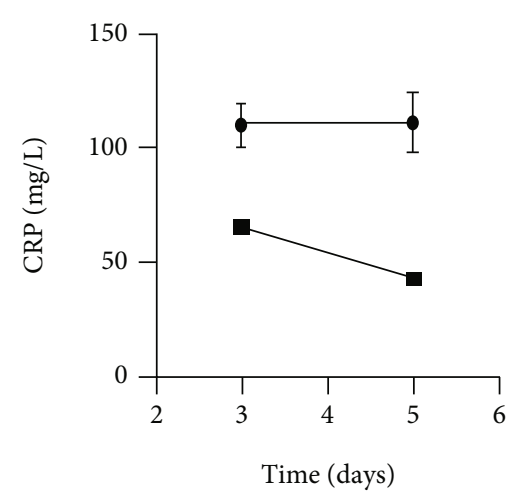

(a)

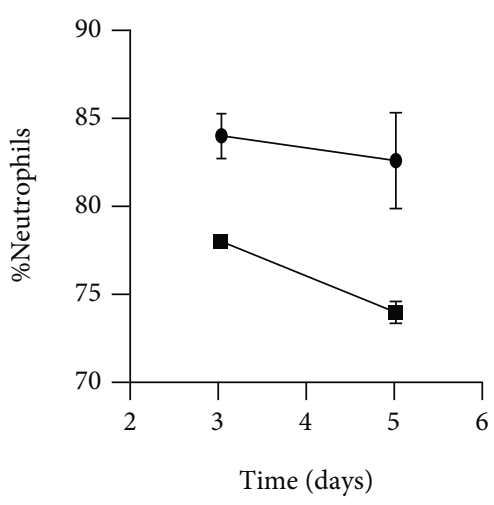

(b)

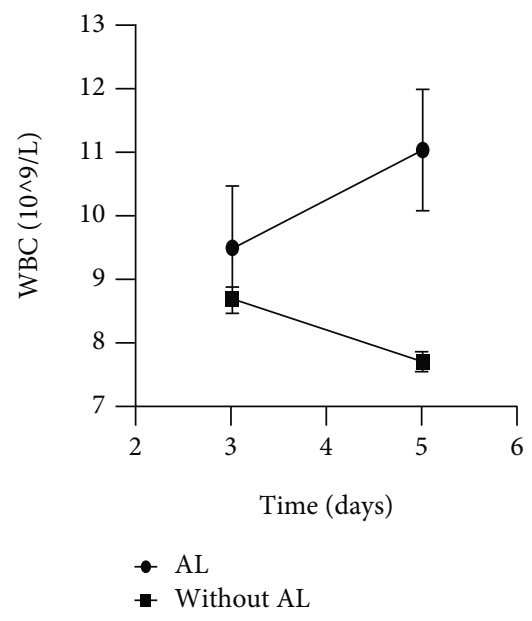

(c)

Figure 1: Mean levels of CRP (a), NEUT (b), and WBC (c) and relative error bars on POD3 and POD5.

TABle 3: Comparison of CAT/MDA/SOD (serum) on POD3 and POD5 in patients with and without anastomotic leak.

\begin{tabular}{lccc}
\hline Parameter $($ mean \pm SD) & AL $(n=16)$ & Without AL $(n=254)$ & $P$ value \\
\hline CAT $_{\text {POD3 }}$ & $45.82 \pm 27.20$ & $86.23 \pm 52.01$ & 0.023 \\
MDA $_{\text {POD3 }}$ & $1.629 \pm 1.119$ & $0.531 \pm 0.309$ & $<0.001$ \\
SOD $_{\text {POD3 }}$ & $0.394 \pm 0.503$ & $0.409 \pm 0.394$ & 0.885 \\
CAT $_{\text {POD5 }}$ & $32.82 \pm 17.21$ & $57.63 \pm 52.26$ & 0.060 \\
MDA $_{\text {POD5 }}$ & $1.912 \pm 1.211$ & $0.419 \pm 0.233$ & $<0.001$ \\
SOD $_{\text {POD5 }}$ & $0.302 \pm 0.218$ & $0.408 \pm 0.127$ & 0.002 \\
\hline
\end{tabular}

CAT: catalase; SOD: superoxide dismutase; MDA: malondialdehyde; POD: postoperative day.

in a contingency table and Fisher's exact test were used. The diagnostic accuracy of tests was quantified using the area (AUC) under the receiver operating characteristic (ROC) curve.

\section{Results}

A total of 270 patients with rectal cancer undergoing elective rectal surgery were enrolled in this study. Of these, 14 patients underwent open rectal resection and 256 underwent laparoscopy surgery. Characteristics of patients are shown in
Table 1. A total of 31 (11.5\%) patients underwent neoadjuvant treatment. Sixteen (5.93\%) patients developed AL. AL was diagnosed between postoperative days 4 and 10. During statistical analyses of AL vs. non-AL, no differences in demographic data except neoadjuvant treatment were observed.

Serum levels of CRP, WBC, and NEUT on POD3 and POD5 are illustrated in Table 2, and their changes are shown in Figure 1. The levels of all three serum factors were higher in the AL group than in the non-AL group and were statistically significant except WBC (POD3). The trends for CRP, WBC, and NEUT in the AL group were basically the same 


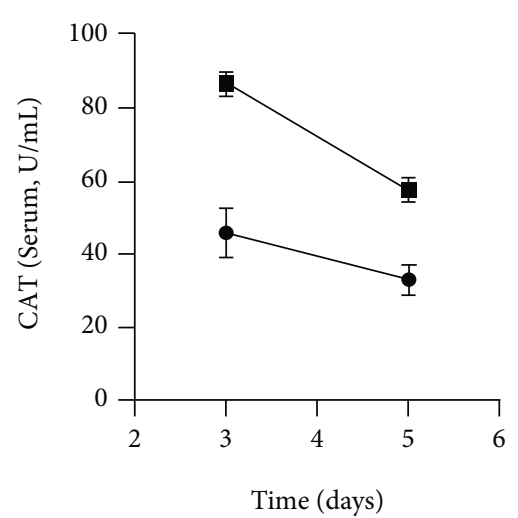

(a)

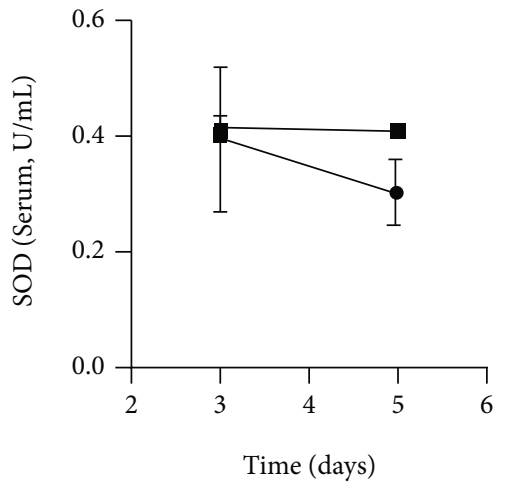

(b)

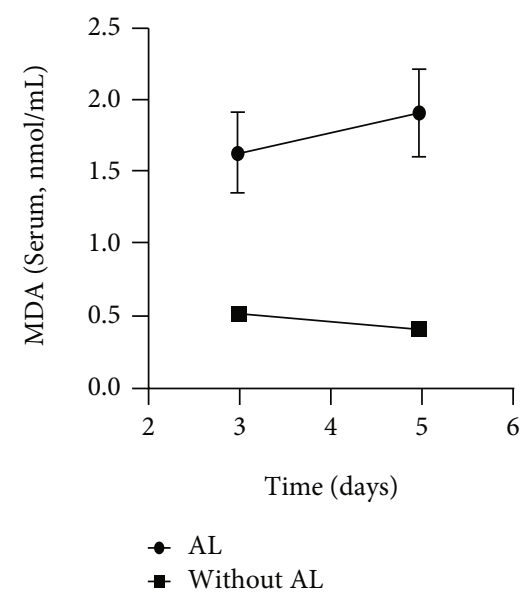

(c)

Figure 2: Mean levels of CAT (a), SOD (b), and MDA (c) and relative error bars on POD3 and POD5 in serum.

TABLE 4: Comparison of CAT/MDA/SOD (drain fluid) on POD3 and POD5 in patients with and without anastomotic leak.

\begin{tabular}{lccc}
\hline $\begin{array}{l}\text { Parameter } \\
(\text { mean } \pm \mathrm{SD})\end{array}$ & $\mathrm{AL}(n=16)$ & $\begin{array}{c}\text { Without } \mathrm{AL} \\
(n=254)\end{array}$ & $\begin{array}{c}P \\
\text { value }\end{array}$ \\
\hline $\mathrm{CAT}_{\text {POD3 }}$ & $59.62 \pm 48.50$ & $90.06 \pm 58.46$ & 0.043 \\
$\mathrm{MDA}_{\text {POD3 }}$ & $0.906 \pm 0.652$ & $0.219 \pm 0.205$ & $<0.001$ \\
$\mathrm{SOD}_{\text {POD3 }}$ & $0.358 \pm 0.112$ & $0.536 \pm 0.237$ & 0.003 \\
$\mathrm{CAT}_{\text {POD5 }}$ & $49.18 \pm 35.68$ & $116.5 \pm 50.91$ & $<0.001$ \\
$\mathrm{MDA}_{\text {POD5 }}$ & $1.387 \pm 0.556$ & $1.294 \pm 0.406$ & 0.840 \\
SOD $_{\text {POD5 }}$ & $0.332 \pm 0.055$ & $0.369 \pm 0.096$ & 0.014 \\
\hline
\end{tabular}

CAT: catalase; SOD: superoxide dismutase; MDA: malondialdehyde; POD: postoperative day.

and increased overall with a slight decrease from POD3 to POD5, while all factors in the non-AL group were lower in comparison.

Serum levels of CAT, MDA, and SOD on POD3 and POD5 are illustrated in Table 3, and their changes are shown in Figure 2. The levels of CAT (POD3) and SOD (POD5) in the AL group were significantly lower than those in the nonAL group while the levels of MDA in the AL group were sig- nificantly higher than those in the non-AL group. Within the AL group between POD3 and POD5, CAT and SOD were decreased and MDA was increased, while in the non-AL group both CAT and MDA were decreased and SOD remained essentially unchanged.

Drain fluid levels of CAT, MDA, and SOD on POD3 and POD5 are illustrated in Table 4, and their changes are shown in Figure 3. The differences in their levels in drainage fluid are similar to those in serum and are statistically significant except for MDA (POD5). Within the AL group between POD3 and POD5, CAT and SOD were decreased and MDA was increased, while in the non-AL group both CAT and MDA were increased and SOD decreased.

All the above indicators with statistically significant differences are drawn with ROC curves (Figures 4-6). The AUC ROC for CRP (POD3), NEUT (POD3), CRP (POD5), NEUT (POD5), and WBC (POD5) are 0.847, $0.779,0.896,0.719$, and 0.766 , respectively. The AUC ROC for CAT (POD3), MDA (POD3), SOD (POD5), and MDA (POD5) in serum are 0.759, 0.831, 0.769, and 0.837, respectively. The AUC ROC for CAT (POD3), MDA (POD3), SOD (POD3), CAT (POD5), and SOD (POD5) in drain fluid are $0.676,0.845,0.738,0.836$, and 0.635 , respectively. 


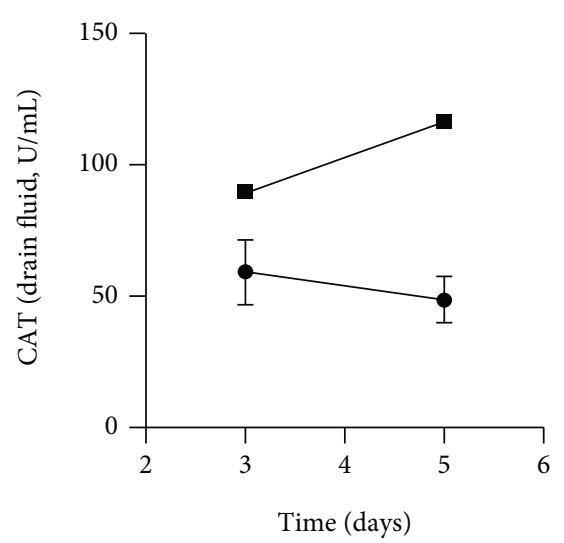

(a)

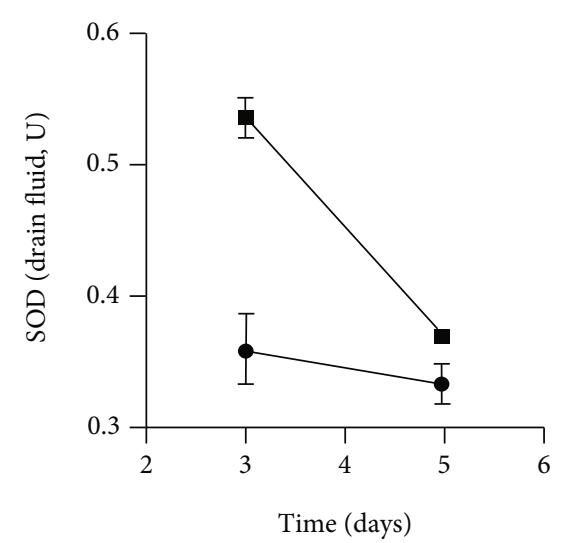

(b)

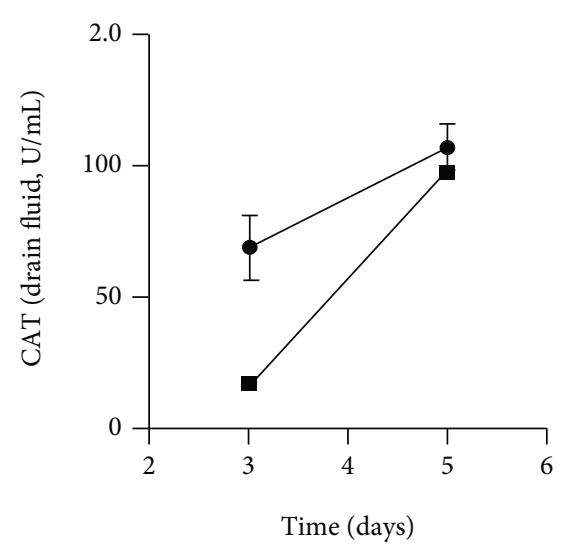

$\rightarrow \mathrm{AL}$

* Without AL

(c)

Figure 3: Mean levels of CAT (a), SOD (b), and MDA (c) and relative error bars on POD3 and POD5 in drain fluid.

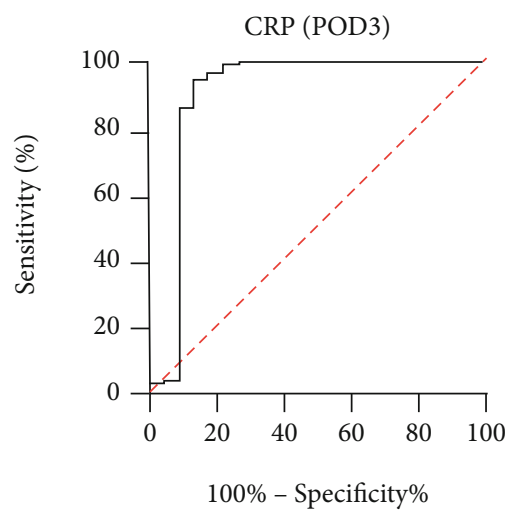

(a)

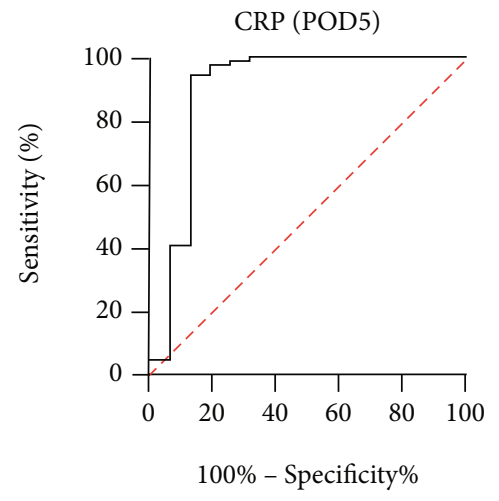

(b)

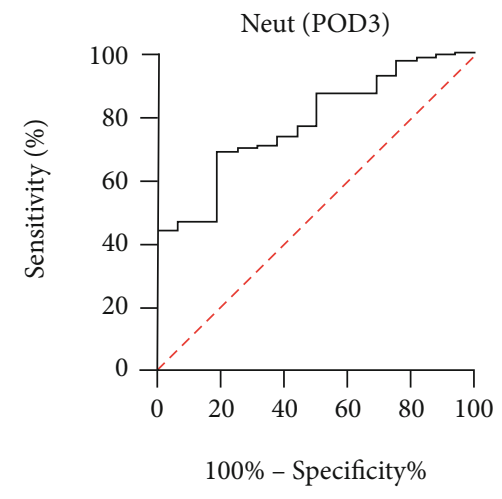

(c)

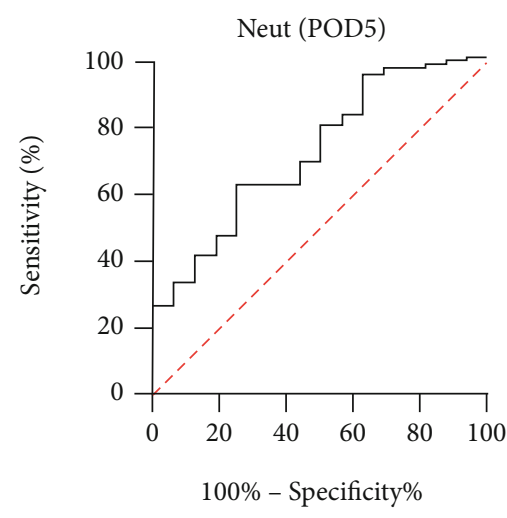

(d)

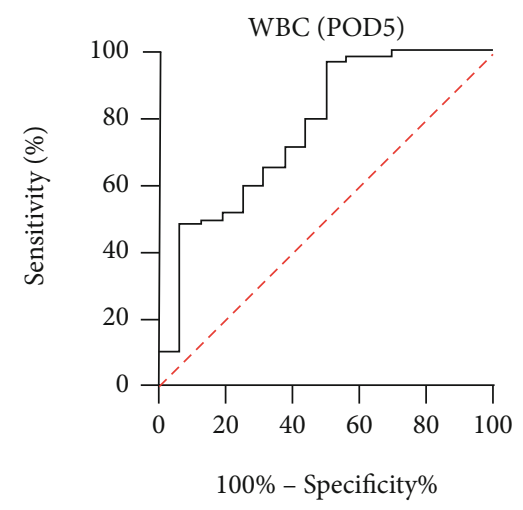

(e)

FIgURE 4: ROC curve analysis on POD3 for CRP (a) and Neut (c) and on POD5 for CRP (b), Neut (d), and WBC (e).

\section{Discussion}

For patients with rectal cancer, surgery is the main treatment method. The surgical method of rectal cancer has gradually developed from the initial partial resection to the expanded radical resection, and recently, it has been moving in the direction of precise and minimally invasive. The surgical method of rectal cancer is constantly being developed and improved. Laparoscopic rectal surgery has become the trend of modern colorectal surgery. Robotic rectal surgery has also been gradually implemented, and the concept of TaTME has also been proposed. With the deepening of the surgeon's 


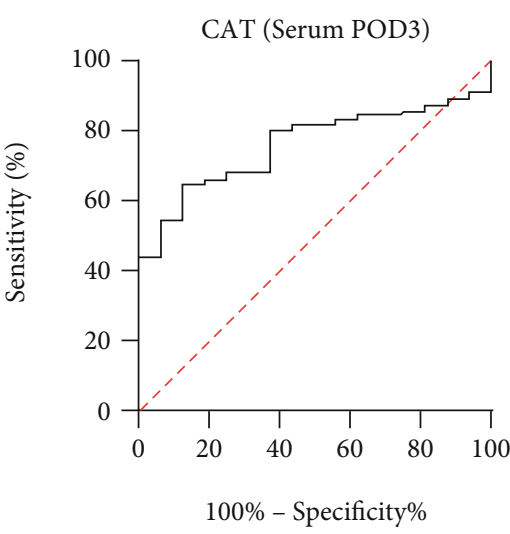

(a)

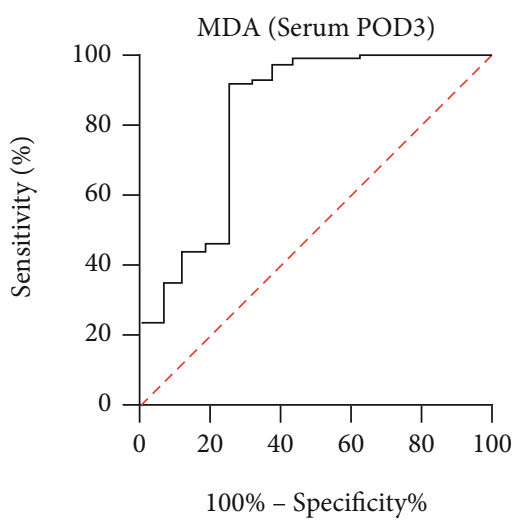

(c)

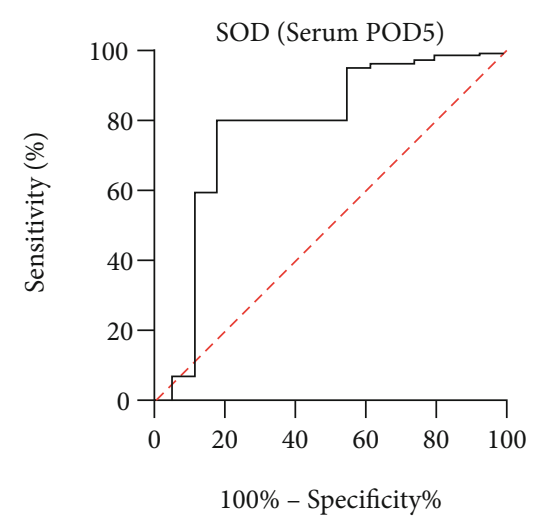

(b)

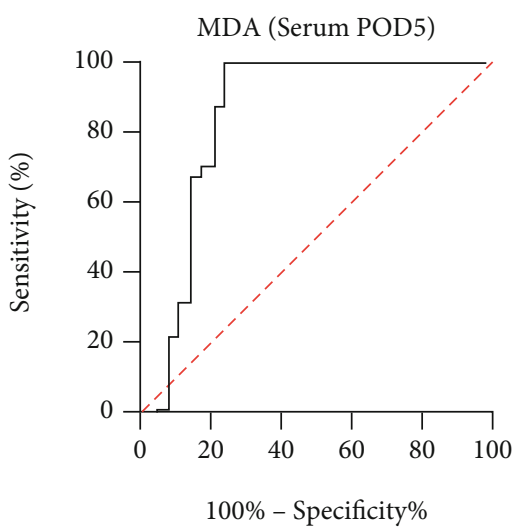

(d)

FIgure 5: ROC curve analysis on POD3 in serum for CAT (a) and MDA (c) and on POD5 for SOD (b) and MDA (d).

understanding of perineum anatomy [20], the probability of complications of nerves and surrounding organs after rectal surgery is decreasing, but the incidence of anastomotic leakage does not seem to be significantly reduced. Compared with open surgery, the incidence of anastomotic leakage is similar in laparoscopic surgery and robotic surgery [21-23]. Early detection of anastomotic leakage is still an urgent problem.

At present, the most widely studied biomarker for prediction of AL is CRP [13, 14, 24-37]. NEUT and WBC counts are also candidate indicators; however, their value in prediction of AL is still controversial [38]. In our research, we first detected three indicators: CRP, NEUT, and WBC on POD3 and POD5, and CRP levels show a predictive effect as reported in the literature (AUC: CRP on POD3 $=0.847$, CPR on POD5 $=0.896$ ).

Whether in the serum or in the drain fluid, the oxidative stress indicators (CAT, MDA, and SOD) we have detected showed a diagnostic value for AL. The level of MDA exhibited the same superior diagnostic accuracy as the level of CRP (AUC: MDA (serum) on POD3 $=0.831$, MDA (serum) on POD5 $=0.837$, and MDA (drain fluid) on POD3 $=0.845$ ).

It is well established that one of the main causes of $\mathrm{AL}$ is a decrease in anastomotic perfusion [39]. Studies have shown that tissue ischemia or necrosis often causes inflammation and oxidative stress, which further damages tissues [40]. The level of oxidative stress may reflect the degree of tissue ischemia. Another factor that is closely related to the healing of anastomosis is the deposition and metabolism of collagen [41]. Matrix metalloproteinases (MMPs) mediate collagen degradation, thereby increasing the risk of AL [42]. It is reported in the literature that oxidative stress injury can upregulate MMP expression, and the level of oxidative stress may reflect the level of MMP expression.

CAT, SOD, and MDA are commonly used oxidative stress indicators. In our study, we observed that compared with those of the non-AL leakage group, the levels of SOD and CAT in the serum and drain fluid of the AL group were significantly reduced, while the level of MDA was significantly increased. Our study found these indicators have a similar AL predictive effect as CRP. SOD and CAT are antioxidant enzymes, which constitute the primary cellular antioxidant defenses [43]. Studies have reported that the rise of SOD, CAT, and other antioxidant enzymes is an indication of tissue repair [44]. MDA has been used as a biomarker of oxidative stress. An increase of MDA reflects the enhancement of lipid peroxidation and tissue damage [45]. We hypothesize that the antioxidant system of patients with anastomosis is impaired, which leads to poor healing of anastomotic tissue. 


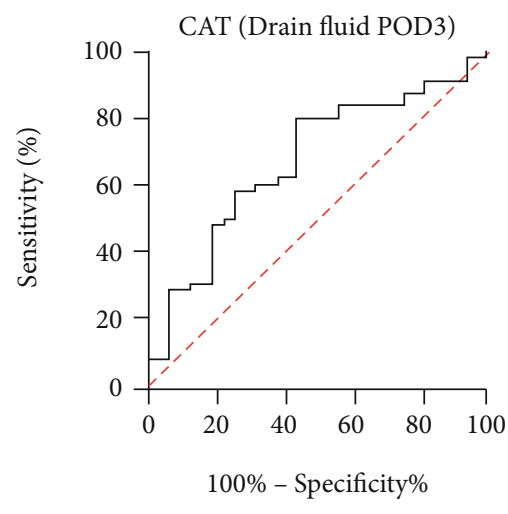

(a)

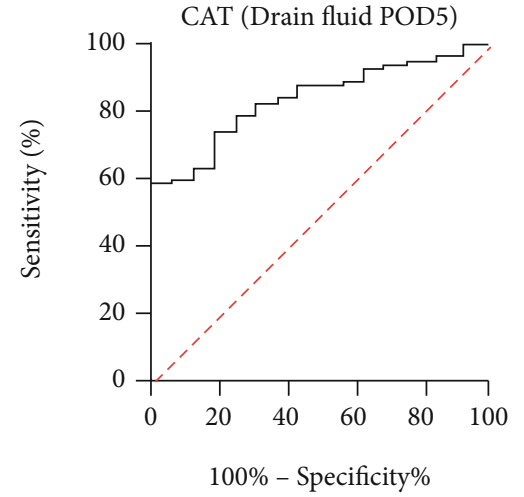

(b)

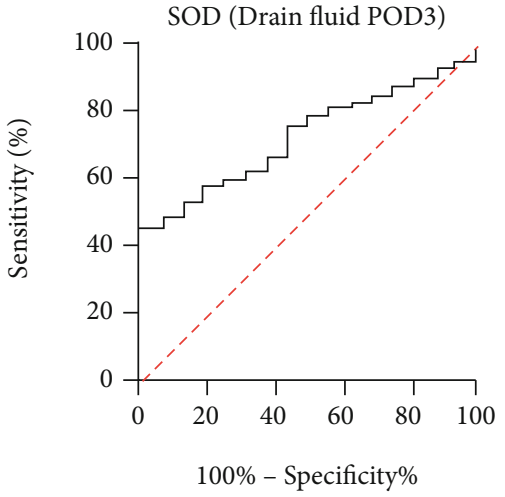

(c)

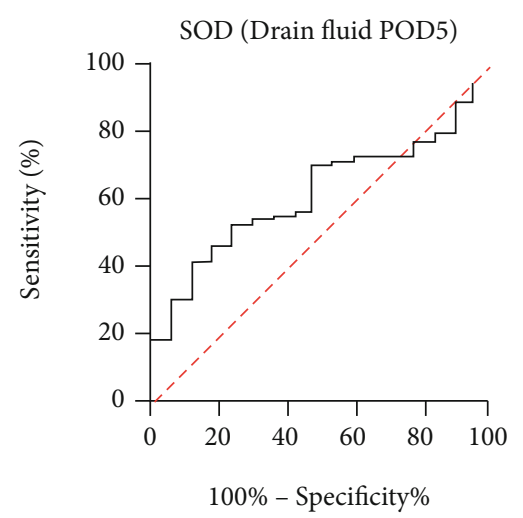

(d)

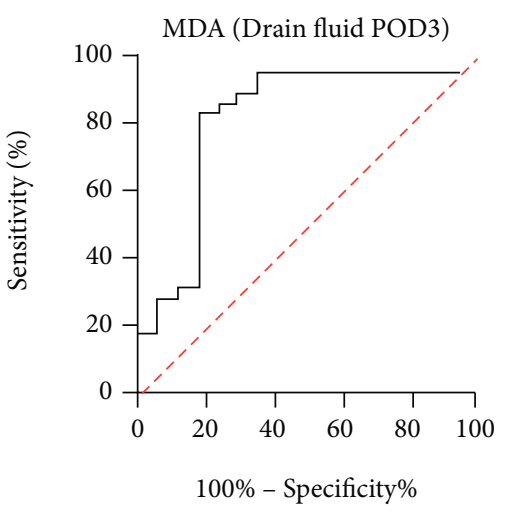

(e)

Figure 6: ROC curve analysis on POD3 in drain fluid for CAT (a), SOD (c), and MDA (e) and on POD5 for CAT (b) and SOD (d).

\section{Conclusions}

In conclusion, the oxidative stress indicators we tested show great potential for the early diagnosis of AL in serum and drain fluid. Our research reveals that the level of MDA in serum or drain fluid has good diagnostic accuracy for AL. The oxidative stress level is expected to become a useful predictor of AL in the future.

\section{Data Availability}

The datasets used and analyzed during the current study are available from the corresponding author on reasonable request.

\section{Conflicts of Interest}

The authors declare that they have no conflicts of interest.

\section{Authors' Contributions}

Qiang Tong designed the study, interpreted the results, and wrote the manuscript. Jiajun Luo, Hongxue $\mathrm{Wu}, \mathrm{Yu}$ Yang, Yue Jiang, and Jingwen Yuan performed the experiments. All authors read and approved the final manuscript. Jiajun Luo and Hongxue Wu contributed equally to this work.

\section{Acknowledgments}

This work was supported by the National Natural Science Foundation of China (No. 81172186 (QT)), by the Natural Science Foundation of Hubei Province (No. 2018CFB504 (QT)), by the Guidance Foundation of Renmin Hospital of Wuhan University (No. RMYD2018M67 (QT)), and by Hubei Province Health and Family Planning Scientific Research Project (No. WJ2019M200 (HXW)).

\section{References}

[1] M. Arnold, M. S. Sierra, M. Laversanne, I. Soerjomataram, A. Jemal, and F. Bray, "Global patterns and trends in colorectal cancer incidence and mortality," Gut, vol. 66, no. 4, pp. 683691, 2017.

[2] M. Rentsch, T. Schiergens, A. Khandoga, and J. Werner, "Surgery for colorectal cancer - trends, developments, and future perspectives," Visceral Medicine, vol. 32, no. 3, pp. 184-191, 2016.

[3] N. C. Buchs, P. Gervaz, M. Secic, P. Bucher, B. Mugnier-Konrad, and P. Morel, "Incidence, consequences, and risk factors for anastomotic dehiscence after colorectal surgery: a prospective monocentric study," International Journal of Colorectal Disease, vol. 23, no. 3, pp. 265-270, 2008.

[4] C. L. Sparreboom, Z. Q. Wu, J. F. Ji, and J. F. Lange, "Integrated approach to colorectal anastomotic leakage: communication, infection and healing disturbances," World Journal of Gastroenterology, vol. 22, no. 32, pp. 7226-7235, 2016. 
[5] Z. R. Lu, N. Rajendran, A. C. Lynch, A. G. Heriot, and S. K. Warrier, "Anastomotic leaks after restorative resections for rectal cancer compromise cancer outcomes and survival," Diseases of the Colon \& Rectum, vol. 59, no. 3, pp. 236-244, 2016.

[6] F. E. Turrentine, C. E. Denlinger, V. B. Simpson et al., "Morbidity, mortality, cost, and survival estimates of gastrointestinal anastomotic leaks," Journal of the American College of Surgeons, vol. 220, no. 2, pp. 195-206, 2015.

[7] J. Meyer, S. Naiken, N. Christou et al., "Reducing anastomotic leak in colorectal surgery: the old dogmas and the new challenges," World Journal of Gastroenterology, vol. 25, no. 34, pp. 5017-5025, 2019.

[8] K. Guyton and J. C. Alverdy, "The gut microbiota and gastrointestinal surgery," Nature Reviews. Gastroenterology \& Hepatology, vol. 14, no. 1, pp. 43-54, 2017.

[9] R. Bachmann, D. Leonard, N. Delzenne, A. Kartheuser, and P. D. Cani, "Novel insight into the role of microbiota in colorectal surgery," Gut, vol. 66, no. 4, pp. 738-749, 2017.

[10] J. V. Cordeiro and A. Jacinto, "The role of transcriptionindependent damage signals in the initiation of epithelial wound healing," Nature Reviews. Molecular Cell Biology, vol. 14, no. 4, pp. 249-262, 2013.

[11] M. Cano Sanchez, S. Lancel, E. Boulanger, and R. Neviere, "Targeting oxidative stress and mitochondrial dysfunction in the treatment of impaired wound healing: a systematic review," Antioxidants, vol. 7, no. 8, p. 98, 2018.

[12] A. Karliczek, N. J. Harlaar, C. J. Zeebregts, T. Wiggers, P. C. Baas, and G. M. van Dam, "Surgeons lack predictive accuracy for anastomotic leakage in gastrointestinal surgery," International Journal of Colorectal Disease, vol. 24, no. 5, pp. 569576, 2009.

[13] E. Asti, G. Bonitta, M. Melloni et al., "Utility of C-reactive protein as predictive biomarker of anastomotic leak after minimally invasive esophagectomy," Langenbeck's Archives of Surgery, vol. 403, no. 2, pp. 235-244, 2018.

[14] J. L. Muñoz, M. O. Alvarez, V. Cuquerella et al., "Procalcitonin and C-reactive protein as early markers of anastomotic leak after laparoscopic colorectal surgery within an enhanced recovery after surgery (ERAS) program," Surgical Endoscopy, vol. 32, no. 9, pp. 4003-4010, 2018.

[15] O. Benoit, M. Faron, N. Margot et al., "C-Reactive protein values after colorectal resection: can we discharge a patient with a C-reactive protein value $>100$ ? A retrospective cohort study," Diseases of the Colon and Rectum, vol. 62, no. 1, pp. 88-96, 2019.

[16] B. A. Messias, R. V. Botelho, S. S. Saad, E. R. Mocchetti, K. C. Turke, and J. Waisberg, "Serum C-reactive protein is a useful marker to exclude anastomotic leakage after colorectal surgery," Scientific Reports, vol. 10, no. 1, p. 1687, 2020.

[17] M. Zawadzki, M. Krzystek-Korpacka, A. Gamian, and W. Witkiewicz, "Serum cytokines in early prediction of anastomotic leakage following low anterior resection," Wideochir Inne Tech Maloinwazyjne, vol. 13, no. 1, pp. 33-43, 2018.

[18] B. Ip, K. T. Ng, S. Packer, S. Paterson-Brown, and G. W. Couper, "High serum lactate as an adjunct in the early prediction of anastomotic leak following oesophagectomy," International Journal of Surgery, vol. 46, pp. 7-10, 2017.

[19] D. A. Clark, T. Cuda, A. Riddell, G. Radford-Smith, and M. Solomon, "Drain fluid amylase as a sensitive biomarker for the early detection of anastomotic leakage in ileal pouch surgery," Colorectal Disease, vol. 21, no. 4, pp. 460-464, 2019.
[20] C. Oikonomou, S. Gourgiotis, R. Cirocchi et al., "Re-exploring the pelvic neuroanatomy from a new perspective and a potential guidance for TaTME: a "bottom-up" approach," Updates in Surgery, vol. 73, no. 2, pp. 503-512, 2021.

[21] S. Di Saverio, W. Stupalkowska, A. Hussein, N. Fearnhead, and J. Wheeler, "Laparoscopic ultralow anterior resection with intracorporeal coloanal stapled anastomosis for low rectal cancer - is robotic surgery or transanal total mesorectal excision always needed to achieve a good oncological and sphinctersparing dissection - a video vignette," Colorectal Disease, vol. 21, no. 7, pp. 848-849, 2019.

[22] C. Staudacher, A. Vignali, D. P. Saverio, O. Elena, and T. Andrea, "Laparoscopic vs. open total mesorectal excision in unselected patients with rectal cancer: impact on early outcome," Diseases of the Colon and Rectum, vol. 50, no. 9, pp. 1324-1331, 2007.

[23] P. Gavriilidis, J. Wheeler, A. Spinelli, N. de'Angelis, C. Simopoulos, and S. di Saverio, "Robotic vs laparoscopic total mesorectal excision for rectal cancers: has a paradigm change occurred? A systematic review by updated metaanalysis," Colorectal Disease, vol. 22, no. 11, pp. 15061517, 2020.

[24] A. B. Almeida, G. Faria, H. Moreira, J. Pinto-de-Sousa, P. Correia-da-Silva, and J. C. Maia, "Elevated serum Creactive protein as a predictive factor for anastomotic leakage in colorectal surgery," International Journal of Surgery, vol. 10, no. 2, pp. 87-91, 2012.

[25] M. Benedetti, P. Ciano, I. Pergolini et al., "Early diagnosis of anastomotic leakage after colorectal surgery by the Dutch leakage score, serum procalcitonin and serum C-reactive protein: study protocol of a prospective multicentre observational study by the Italian ColoRectal Anastomotic Leakage (iC)," Il Giornale di Chirurgia, vol. 40, no. 1, pp. 20-25, 2019.

[26] Y. M. Ho, J. Laycock, A. Kirubakaran, L. Hussain, and J. Clark, "Systematic use of the serum C-reactive protein concentration and computed tomography for the detection of intestinal anastomotic leaks," ANZ Journal of Surgery, vol. 90, no. 1-2, pp. 109-112, 2020.

[27] N. Lagoutte, O. Facy, A. Ravoire et al., "C-reactive protein and procalcitonin for the early detection of anastomotic leakage after elective colorectal surgery: Pilot study in 100 patients," Journal of Visceral Surgery, vol. 149, no. 5, pp. e345-e349, 2012.

[28] J. Lu, L. Zheng, R. Li et al., "Diagnostic value of dynamic monitoring of C-reactive protein in drain drainage to predict early anastomotic leakage after colorectal cancer surgery," Zhonghua Wei Chang Wai Ke Za Zhi, vol. 20, no. 9, pp. 10551059, 2017.

[29] P. Matthiessen, M. Henriksson, O. Hallböök, E. Grunditz, B. Norén, and G. Arbman, "Increase of serum C-reactive protein is an early indicator of subsequent symptomatic anastomotic leakage after anterior resection," Colorectal Disease, vol. 10, no. 1, pp. 75-80, 2008.

[30] I. Mintziras, E. Maurer, V. Kanngiesser, and D. K. Bartsch, "Creactive protein and drain amylase accurately predict clinically relevant pancreatic fistula after partial pancreaticoduodenectomy," International Journal of Surgery, vol. 76, pp. 53-58, 2020.

[31] P. Ortega-Deballon, F. Radais, O. Facy et al., "C-Reactive protein is an early predictor of septic complications after elective colorectal surgery," World Journal of Surgery, vol. 34, no. 4, pp. 808-814, 2010. 
[32] J. J. Platt, M. L. Ramanathan, R. A. Crosbie et al., "C-reactive protein as a predictor of postoperative infective complications after curative resection in patients with colorectal cancer," Annals of Surgical Oncology, vol. 19, no. 13, pp. 4168-4177, 2012.

[33] I. S. Reynolds, M. R. Boland, F. Reilly et al., "C-reactive protein as a predictor of anastomotic leak in the first week after anterior resection for rectal cancer," Colorectal Disease, vol. 19, no. 9, pp. 812-818, 2017.

[34] P. P. Singh, I. S. Zeng, S. Srinivasa, D. P. Lemanu, A. B. Connolly, and A. G. Hill, "Systematic review and meta-analysis of use of serum C-reactive protein levels to predict anastomotic leak after colorectal surgery," The British Journal of Surgery, vol. 101, no. 4, pp. 339-346, 2014.

[35] S. R. Smith, P. Pockney, R. Holmes et al., "Biomarkers and anastomotic leakage in colorectal surgery: C-reactive protein trajectory is the gold standard," ANZ Journal of Surgery, vol. 88, no. 5, pp. 440-444, 2018.

[36] P. Waterland, J. Ng, A. Jones et al., "Using CRP to predict anastomotic leakage after open and laparoscopic colorectal surgery: is there a difference?," International Journal of Colorectal Disease, vol. 31, no. 4, pp. 861-868, 2016.

[37] T. Welsch, S. A. Müller, A. Ulrich et al., "C-reactive protein as early predictor for infectious postoperative complications in rectal surgery," International Journal of Colorectal Disease, vol. 22, no. 12, pp. 1499-1507, 2007.

[38] A. T. Stearns, F. Liccardo, K. N. Tan et al., "Physiological changes after colorectal surgery suggest that anastomotic leakage is an early event: a retrospective cohort study," Colorectal Disease, vol. 21, no. 3, pp. 297-306, 2019.

[39] C. Zhang, L. Chen, M. Cui et al., "Short- and long-term outcomes of rectal cancer patients with high or improved low ligation of the inferior mesenteric artery," Scientific Reports, vol. 10, no. 1, article 15339, 2020.

[40] P. H. Sung, K. C. Lin, H. T. Chai et al., "Losing regulation of the extracellular matrix is strongly predictive of unfavorable prognostic outcome after acute myocardial infarction," International Journal of Molecular Sciences, vol. 21, no. 17, article 6219, 2020.

[41] X. H. Wang, J. S. Ni, N. L. Cao et al., "In vivo evaluation of Mg$6 \mathrm{Zn}$ and titanium alloys on collagen metabolism in the healing of intestinal anastomosis," Scientific Reports, vol. 7, no. 1, article 44919, 2017.

[42] P. M. Krarup, M. Eld, K. Heinemeier, L. N. Jorgensen, M. B. Hansen, and M. S. Ågren, "Expression and inhibition of matrix metalloproteinase (MMP)-8, MMP-9 and MMP-12 in early colonic anastomotic repair," International Journal of Colorectal Disease, vol. 28, no. 8, pp. 1151-1159, 2013.

[43] B. Qian, J. Li, K. Guo et al., "Antioxidant biocompatible composite collagen dressing for diabetic wound healing in rat model," Regenerative Biomaterials, vol. 8, no. 2, article rbab003, 2021.

[44] R. Melekoglu, O. Ciftci, S. Eraslan, A. Cetin, and N. Basak, "Beneficial effects of curcumin and capsaicin on cyclophosphamide-induced premature ovarian failure in a rat model," Journal of Ovarian Research, vol. 11, no. 1, p. 33, 2018.

[45] M. I. Ezzat, M. M. Okba, S. H. Ahmed et al., "In-depth hepatoprotective mechanistic study of Phyllanthus niruri: in vitro and in vivo studies and its chemical characterization," PLOS One, vol. 15, no. 1, article e0226185, 2020. 